\title{
104. On Singular Perturbation of Linear Partial Differential Equations with Constant Coefficients. I
}

\author{
By Mitio Nagumo
}

(Comm. by K. KunUGi, M.J.A., Oct. 12, 1959)

1. Introduction. Let $(t, x)=\left(t, x_{1}, \cdots, x_{m}\right)$ be $m+1$ real variables in $t \geqq 0, x \in E^{m}$, where $E^{m}$ denotes the $m$-dimensional Euclidean space. Let $L_{\mathrm{s}}$ be an $r \times r$ matrix of differential operators with constant coefficients depending on a parameter $\varepsilon$

$$
L_{c}=\sum_{j=1}^{l} P_{j}\left(\partial_{x}, \varepsilon\right) \partial_{t}^{j 1)}
$$

where $P_{j}(\xi, \varepsilon)$ are $r \times r$ matrices of polynomials in $\xi=\left(\xi_{1}, \cdots, \xi_{m}\right)$, whose coefficients depends on $\varepsilon \geqq 0$ continuously, and let us consider a system of partial differential equations

$$
L_{\varepsilon}[u]=f(t, x, \varepsilon),
$$

where $u=\left(u_{\rho} \rho \downarrow 1, \cdots, r\right) f=\left(f_{\rho} \rho \downarrow 1, \cdots, r\right){ }^{2)} \quad$ We assume that $P_{l}(\xi, \varepsilon)$ $=P_{l}(\varepsilon)$ does not contain $\xi$ and

$$
\operatorname{det}\left(P_{l}(\varepsilon)\right) \neq 0 \text { for } \varepsilon>0 \text {. }
$$

In this note we are concerned with showing the relationship of (1), as $\varepsilon \downarrow 0$, to a particular solution of a related system (for $\varepsilon=0$ )

$$
L_{0}[u]=f(t, x, 0) \text {, }
$$

especially when $L_{0}$ is degenerated, i.e.

$$
\operatorname{det}\left(P_{l}(0)\right)=0 . .^{3)}
$$

Let $C_{0}^{\infty}$ be the set of all on $E^{m}$ infinite times continuously differentiable complex valued functions with compact carrier. For any $u \in C_{0}^{\infty}$ we define the norm $\|u\|_{p}$ by

$$
\|u\|_{p}^{2}=\int_{\mathbb{E}^{m}} \sum_{|\nu| \leq p}\left|\partial_{1}^{\nu_{1}} \cdots \partial_{m}^{\nu_{m}} u(x)\right|^{2} d x,,^{4)} \quad\left(|\nu|=\nu_{1}+\cdots+\nu_{m}\right) .
$$

The completion of $C_{0}^{\infty}$ with respect to the norm (3) will be denoted by $H_{p} . \quad H_{p}$ is a kind of Hilbert space. One sees easily

$$
H_{p} \supset H_{p^{\prime}} \text { and }\|u\|_{p} \leqq\|u\|_{p^{\prime}} \text { if } p<p^{\prime} .
$$

We set $H_{\infty}=\bigcap_{p<\infty} H_{p}$, then $H_{\infty}$ is a linear topological space with a sequence of semi-norms $\|u\|_{p}(p=0,1,2, \cdots)$ for $u \in H_{\infty} . \quad H_{\infty}$ is dense in $H_{p}$ for any $p$, and $C_{0}^{\infty}$ is dense in $H_{\infty}$ (hence in $H_{p}$ ).

Let $\hat{\varphi}$ be the Fourier transform of $\varphi \in H_{p}$,

$$
\widehat{\varphi}(\xi)=\frac{1}{\sqrt{2 \pi}^{m}} \int_{E^{m}} e^{-i \xi \cdot x} \varphi(x) d x=\mathscr{F}[\varphi],
$$

1) We use $\partial_{t}$ for $\partial / \partial_{t}$, and $\partial_{x}$ for $\partial / \partial x_{1}, \cdots, \partial / \partial x_{m}$.

2) ( $\left.u_{\rho} \rho \downarrow 1, \cdots, r\right)$ means the $r$-dimensional vector (column) with the components $\left(u_{1}, \cdots, u_{r}\right)$.

3) The condition (2) is not essential in the general consideration.

4) $\partial_{\mu}$ is the abbreviation of $\partial / \partial x_{\mu}$. 
then $\varphi \in H_{p}$ is equivalent to $\left(1+|\xi|^{2}\right)^{p / 2} \hat{\varphi}(\xi) \in L^{2}$ and

$$
\|\varphi\|_{p}^{2}=\int_{E^{m}}\left(1+|\xi|^{2}\right)^{p}|\hat{\varphi}(\xi)|^{2} d \xi=\|\hat{\varphi}\|_{p}^{\prime 2} .
$$

The complete space of all measurable complex valued functions $\hat{\varphi}$ such that $\|\hat{\varphi}\|_{p}^{\prime}<\infty$ will be denoted by $\hat{H}_{p}{ }^{5}$ ) The Fourier transform $\widetilde{\mho}$ is a unitary transformation of $H_{p}$ onto $\hat{H}_{p}$.

For any real number $p \gtrless 0$, we can define the norm $\|\varphi\|_{p}$ for $\varphi \in C_{0}^{\infty}$ by (5). If $p \geqq 0$, then the completion of $C_{0}^{\infty}$ which we denote by $H_{p}$, with respect to the norm (5) is the set of all complex valued measurable functions such that $\|\varphi\|_{p}<\infty .^{5)}$ But if $p<0$, the completion of $C_{0}^{\infty}$ with respect to (5) consists from a class of distributions by L. Schwarz. The Fourier transform of $H_{p}$, denoted by $\hat{H}_{p}$, even if $p<0$, is the set of all measurable functions $\hat{\varphi}^{5)}$ such that $\|\hat{\varphi}\|_{p}^{\prime}<\infty$ by (5).

Let $D^{(k)}$ be any differential operator with constant coefficients of order $k$, then $D^{(k)}$ is a bounded linear mapping of $H_{p}$ into $H_{p-k}$.

Let $F_{x}$ be any linear functional space, whose elements are functions of $x \in E^{m}$, and $\varphi(t)$ be a variable element of $F_{x}$ depending on a real parameter $t$ in an interval $J$. We say " $\varphi(t)$ is $F_{x}$-continuous in $t \in J$ " if the mapping $t \in J \longrightarrow \varphi(t) \in F_{x}$ is continuous, and " $\varphi(t)$ is $F_{x}$-differentiable at $t=t_{0}$ " if

$$
\left(t-t_{0}\right)^{-1}\left\{\varphi(t)-\varphi\left(t_{0}\right)\right\} \rightarrow \varphi^{\prime}\left(t_{0}\right) \text { in } F_{x} \text { as } t \rightarrow t_{0} .
$$

We use the notation $\varphi^{\prime}(t)=\frac{d}{d t} \varphi(t)$, if $\varphi^{\prime}(t)$ defined by (6) has meaning

for $t$ in an interval. If $D^{(k)}$ is a differential operator in $x \in E^{m}$ with constant coefficients of order $k$ and $\varphi(t)$ is $H_{p, x}$-continuous in $t$, then $D^{(k)} \varphi(t)$ is $H_{p-k, x}$-continuous, and if $\varphi(t)$ is $H_{p, x}$-differentiable in $t$ then $D^{(k)} \varphi(t)$ is $H_{p-k, x}$-differentiable in $t$ and

$$
\frac{d}{d t}\left\{D^{(k)} \varphi(t)\right\}=D^{(k)}\left\{\frac{d}{d t} \varphi(t)\right\} \text {. }
$$

Let $u=u(t)=u(t, x)$ be $l$ times continuously $H_{p, x}$-differentiable in $t \in J$, and $L$ be a differential operator in $(t, x)$ with constant coefficients defined by

$$
L[u]=\sum_{j=0}^{l} P_{j}\left(\partial_{x}\right) \partial_{t}^{j} u(t, x),
$$

where $P_{j}(\xi)$ are polynomials in $\xi=\left(\xi_{1}, \cdots, \xi_{m}\right)$ of degree at most $k$ with constant coefficients. Then $L[u](t)$ is $H_{p-k, x}$-continuous in $t \in J$. Putting (8)

$$
L[u](t)=f(t)
$$

we say $u(t)$ is an $H_{p}$-solution of the equation (8).

Now we extend the operator $L$ as follows:

5) Strictly speaking, each element of the space is a such class of functions, that any pair of which differ at most on a set of measure zero. 
Definition 1. Let $\left\{u_{\nu}(t)\right\}_{\nu=1}^{\infty}\left(u_{\nu}(t)=u_{\nu}(t, x)\right)$ be a sequence of $l$ times continuously $H_{p, x}$-differentiable functions in $t \in J$, such that as $\nu \rightarrow \infty, u_{\nu}(t) \rightarrow u(t)$ in $H_{p, x}$ quasi-uniformly for $t \in J,{ }^{6)}$ and $L\left[u_{\nu}(t)\right]$ $\rightarrow f(t)$ in $H_{p-k, x}$ quasi-uniformly for $t \in J$. Then we define $L[u(t)]$ $=f(t)$ for $t \in J$, and we say $u(t)$ is a generalized $\boldsymbol{H}_{\boldsymbol{p}}$-solution of (8).

A generalized $H_{p}$-solution is naturally $H_{p, x}$-continuous in $t$, but it is not necessarily $H_{p, x}$-differentiable in $t$. This extension of the operator $L$ is free from absurdity. Because, $L$ is a pre-closed linear operator as follows:

If $u_{\nu}(t) \rightarrow 0$ in $H_{p, x}$ quasi-uniformly for $t \in J$, and $L\left[u_{\nu}(t)\right] \rightarrow f(t)$ in $H_{p-k, x}$ quasi-uniformly for $t \in J$, then $f(t)=0$ for $t \in J$.

We say "a system $u_{1}(t), \cdots, u_{r}(t)$ has property $(P)$ " if each $u_{\rho}(t)$ $(\rho=1, \cdots, r)$ has the property $(P)$. The above definitions and related statements can be all extended to a system of functions and system of operators in a quite similar way, so that we need not explain them in detail.

2. Preliminary theorems. In the following let us give some preliminary theorems without proof.

Let $L$ be a matrix of differential operators

$$
L=\sum_{j=1}^{l} P_{j}\left(\partial_{x}\right) \partial_{t}^{j}
$$

where $P_{j}(\xi)$ are $r \times r$ matrices of polynomials in $\xi=\left(\xi_{1}, \cdots, \xi_{m}\right)$ at most of order $k$ with constant coefficients, and $P_{l}(\xi)=P_{l}$ be a constant matrix such that $\operatorname{det}\left(P_{l}\right) \neq 0$.

Theorem 1. If $u=u(t)=u(t, x)$ is a generalized $H_{p}$-solution of $L[u]=f(t)$ for $t \in J$, then there exists a sequence of $l$ times continuously $C_{0, x}^{\infty}$-differentiable $u_{\nu}(t)=u_{\nu}(t, x)$ for $t \in J$, such that as $\nu \rightarrow \infty, u_{\nu}(t) \rightarrow$ $u(t)$ in $H_{p, x}$ quasi-uniformly for $t \in J$, and $L\left[u_{\nu}(t)\right] \rightarrow f(t)$ in $H_{p-k, x}$ quasi-uniformly for $t \in J$.

We associate the partial differential equation $L[u]=f(t)$ with the following ordinary differential equation

$$
\sum_{\mu=0}^{l} P_{\mu}(i \xi)\left(\frac{d}{d t}\right)^{\mu} Y=0 .
$$

Let $Y_{j}(t, \xi)$ be matricial solutions of $(2.1)$ with the initial conditions

$$
\left(\partial_{t}^{k-1} Y\right)_{t=0}=\delta_{j k} 1 \text {. }
$$

Theorem 2. If there exist constants $C$ and $q$ such that

$$
\left|Y_{j}(t, \xi)\right|^{7)} \leqq C \sqrt{1+|\xi|^{2}}(j=1, \cdots, l) \text { for } 0 \leqq t \leqq T
$$

and $f(t, x)$ is $H_{p, x}$-continuous in $0 \leqq t \leqq T$, then the partial differential equation

$$
L[u]=\sum_{\mu=0}^{l} P_{\mu}\left(\partial_{x}\right) \partial_{t}^{\mu} u=f(t, x)
$$

6) "Quasi-uniform for $t \in J$ " means " uniform for any compact part of $J$ ".

7) $|Y|$ is the norm of the matrix $Y$, defined by $|Y|=\operatorname{Sup}_{u \neq 0}\{|Y u| /|u|\}$. 
has generalized $H_{p-q}$-solution $u=u(t, x)$ with the initial conditions

$$
\partial_{t}^{j-1} u(0, x)=\varphi_{j}(x) \quad(j=1, \cdots, l),
$$

where $\varphi_{j}$ are arbitrary functions of $H_{p, x}$. Further if $p \geqq q$, then this solution $u=u(t, x)$ is represented by

$$
\begin{gathered}
u(t, x)=\sum_{j=1}^{l} \frac{1}{\sqrt{2 \pi}} \int_{E^{m}} e^{i x \cdot \xi} Y_{j}(t, \xi) \widehat{\varphi}_{j}(\xi) d \xi \\
+\frac{1}{\sqrt{2 \pi}^{m}} \int_{E^{m}} e^{i x \cdot \xi} d \xi \int_{0}^{t} P_{l}^{-1} Y_{l}(t-\tau, \xi) \hat{f}(\tau, \xi) d t,
\end{gathered}
$$

where $\hat{\varphi}_{j}$ and $\hat{f}$ are Fourier transforms of $\varphi_{j}$ and $f$ respectively as functions of $x$.

Further if

$$
\left|\partial_{t}^{k-1} Y_{j}(t, \xi)\right|<C{\sqrt{1+|\xi|^{2}}}^{q} \text { for } 0 \leqq t \leqq T,
$$

$k=1, \cdots, l, j=1, \cdots, l$, then the solution $u=u(t, x)$ is an $H_{p-q}$-solution in proper sense.

3. Stability. Consider a system of equations containing a parameter $\varepsilon$

$$
L_{\varepsilon}[u]=\sum_{\mu=0}^{l} P_{\mu}\left(\partial_{x}, \varepsilon\right) \partial_{t}^{\mu} u=f_{\varepsilon}(t, x),
$$

where $P_{\mu}(\xi, \varepsilon)$ are $r \times r$ matrices of polynomials in $\xi=\left(\xi_{1}, \cdots, \xi_{m}\right)$ with constant coefficients depending on $\varepsilon$ continuously for $\varepsilon \geqq 0$, and $P_{l}(\varepsilon)=$ $P_{l}(\xi, \varepsilon)$ depends on $\varepsilon$ only and

$$
\operatorname{det}\left(P_{l}(\varepsilon)\right) \neq 0 \text { for } \varepsilon>0 \text {. }
$$

Definition 2. We say that the equation (3.1) is $\mathbf{H}_{\boldsymbol{p}}$-stable for $\varepsilon \downarrow 0$ in $0 \leqq t \leqq T$ with respect to a particular solution $u=u_{0}(t)$ of (3.1) for $\varepsilon=0$, if and only if,

whenever

$$
u_{s}(t) \rightarrow u_{0}(t) \text { in } H_{p, x} \text { uniformly for } 0 \leqq t \leqq T,
$$

(3.2) $f_{c}(t)=f_{\odot}(t, x) \rightarrow f_{0}(t)$ in $H_{p, x}$ uniformly for $0 \leqq t \leqq T$, and $u_{s}(t)=u(t, x, \varepsilon)$ is a generalized $H_{p}$-solution of (3.1) such that

$$
\partial_{t}^{j-1} u_{s}(0) \rightarrow \partial_{t}^{j-1} u_{0}(0) \text { in } H_{p, x}(j=1, \cdots, l) .
$$

Theorem 3. Let degree of $\left\{P_{\mu}(\xi, \varepsilon)-P_{\mu}(\xi, 0)\right\}=k(\mu=0, \cdots, l)$, and let $u=u_{0}(t)$ be an $l$ times continuously $H_{p+k, x}$-differentiable solution of (3.1) for $\varepsilon=0$ in $0 \leqq t \leqq T$. In order that (3.1) be $H_{p}$-stable for $\varepsilon \downarrow 0$ with respect to $u=u_{0}(t)$ in $0 \leqq t \leqq T$, it is necessary and sufficient that, there exist constants $\varepsilon_{0}>0$ and $C$ such that

$$
\operatorname{Sup}_{\xi \in E^{m}}\left|Y_{j}(t, \xi, \varepsilon)\right| \leqq C \text { for } 0 \leqq t \leqq T, 0<\varepsilon \leqq \varepsilon_{0},
$$

$$
\operatorname{Sup}_{\xi \in E^{m}} \int_{0}^{T}\left|P_{l}(\varepsilon)^{-1} Y_{l}(t, \xi, \varepsilon)\right| d t \leqq C \text { for } 0<\varepsilon \leqq \varepsilon_{0},
$$

where $y=Y_{j}(t, \xi, \varepsilon)$ are matricial solutions of

$$
\sum_{\mu=0}^{l} P_{\mu}(i \xi, \varepsilon)\left(\frac{d}{d t}\right)^{\mu} y=0
$$


with the initial conditions $\partial_{t}^{k-1} Y_{j}(0, \xi, \varepsilon)=\delta_{k j} 1 \quad(k=1, \cdots, l)$.

Proof. Necessity of (3.4): Let $v=v_{c}(t)$ be the solution of

$$
L_{\varepsilon}[v]=0
$$

with the initial conditions $\partial_{t}^{j-1} v_{s}(0)=\partial_{t}^{j-1} u_{\varepsilon}(0)-\partial_{t}^{j-1} u_{0}(0) \quad(j=1, \cdots, l)$. One sees easily, it is necessary that

$$
v_{s}(t) \rightarrow 0 \text { in } H_{p, x} \text { uniformly for } 0 \leqq t \leqq T .
$$

Now assume that for any $\varepsilon_{0}>0$, there did not exist such $C$ that (3.4) holds. Then, for a certain $j$, there are sequences $\left\{\varepsilon_{\nu}\right\}$ and $\left\{t_{\nu}\right\}$ such that, $\varepsilon_{\nu} \downarrow 0$ as $\nu \rightarrow \infty, 0 \leqq t_{\nu} \leqq T$, and a sequence of spheres $\left\{S_{\nu}\right\}, S_{\nu}=$ $\left\{\xi ;\left|\xi-\xi^{(\nu)}\right|<\delta_{\nu}\right\}$, such that

We set

$$
\begin{aligned}
& \left|Y_{j}\left(t_{\nu}, \xi, \varepsilon_{\nu}\right)\right|>\nu \quad \text { for } \xi \in S_{\nu}, \\
& 2^{-1}<{\sqrt{1+|\xi|^{2}}}^{p} /{\sqrt{1+\left|\xi^{(\nu)}\right|^{2}}}^{p}<2 \text { for } \xi \in S_{\nu} \text {. }
\end{aligned}
$$

$$
v_{\nu}(t, x)=\frac{\alpha_{\nu}}{\sqrt{2 \pi}^{m}} \int_{S_{\nu}} e^{i x \cdot \xi} Y_{j}\left(t, \xi, \varepsilon_{\nu}\right) d \xi,
$$

with $\alpha_{\nu}=\left(\text { measure of } S_{\nu}\right)^{-1}{\sqrt{1+\left|\xi^{(\nu)}\right|^{2}}}^{-p}$. Then $v=v_{\nu}(t, x)$ is an $H_{\infty}$ solution of (3.7) such that, by (3.9),

$$
\left\|\partial_{t}^{j-1} v_{\nu}(0)\right\|_{p} \leqq 2 \nu^{-1} \rightarrow 0, \quad \partial_{t}^{k-1} v_{\nu}(0)=0 \text { for } k \neq j,
$$

and $\left\|v_{\nu}\left(t_{\nu}\right)\right\|_{p} \geqq 1 / 2$. This contradicts with (3.8). The condition (3.4) is thus necessary.

Necessity of (3.5): If (3.5) did not hold for any $\varepsilon_{0}>0$ and $C$, then there would exist a sequence $\left\{\varepsilon_{\nu}\right\}, \varepsilon_{\nu} \downarrow 0$ and a sequence of spheres $\left\{S_{\nu}\right\} \subset E^{m}$ such that

$$
\int_{0}^{T}\left|P_{l}\left(\varepsilon_{\nu}\right)^{-1} Y_{l}\left(T-\tau, \xi, \varepsilon_{\nu}\right)\right| d \tau>\nu \text { for } \xi \in S_{\nu}
$$

Let $u=u_{\nu}(t)=u_{\nu}(t, x)$ be generalized $H_{p}$-solution of (3.1) with the initial conditions $\partial_{t}^{j-1} u_{\nu}(0)=\hat{o}_{t}^{j-1} u_{0}(0) \quad(j=1, \cdots, l)$. Then $v=v_{\nu}(t)=u_{\nu}(t)-u_{0}(t)$ is a generalized $H_{p}$-solution of

$$
L_{s_{\nu}}[u]=g_{\nu}(t)
$$

with $g_{\nu}(t)=g_{\nu}(t, x)=\left\{L_{s_{\nu}}-L_{0}\right\}\left[u_{0}\right]+f_{s_{\nu}}(t)-f_{0}(t)$, with the initial conditions $\partial_{t}^{j-1} v_{\nu}(0)=0 \quad(j=1, \cdots, l)$. By Theorem 2, since $g_{\nu}(t)$ is $H_{p, x}$-continuous and (3.4) holds,

$$
v_{\nu}(t, x)=\frac{1}{\sqrt{2 \pi}} \int_{E^{m}} e^{i x \cdot \xi}\left\{\int_{0}^{t} P_{l}(\varepsilon)^{-1} Y_{l}\left(t-\tau, \xi, \varepsilon_{\nu}\right) \hat{g}_{\nu}(\tau, \xi) d \tau\right\} d \xi
$$

where $\hat{g}_{\nu}(t, \xi)$ denotes the Fourier transform of $g_{\nu}(t, x)$ as the function of $x$. Since $\left\{L_{s_{\nu}}-L_{0}\right\}\left[u_{0}\right]$ is $H_{p, x}$-continuous and

$$
\left\{L_{c_{\nu}}-L_{0}\right\}\left[u_{0}\right] \rightarrow 0 \text { in } H_{p, x} \text { uniformly for } 0 \leqq t \leqq T,
$$

$g_{\nu}(t)$ may be any $H_{p, x}$-continuous function such that $g_{\nu}(t) \rightarrow 0$ in $H_{p, x}$ uniformly for $0 \leqq t \leqq T$.

Now by $(3.10)$ we can find a continuous function $\psi_{\nu}(t, \xi)$ in $0 \leqq t \leqq T$, $\xi \in S_{\nu}$, such that 
(3.13) $\left\{\begin{array}{c}\left|\psi_{\nu}(t, \xi)\right| \leqq 1, \\ \left|\int_{0}^{T} P_{l}\left(\varepsilon_{\nu}\right)^{-1} Y_{l}\left(T-\tau, \xi, \varepsilon_{\nu}\right) \psi_{\nu}(\tau, \xi) d \tau\right|>\nu \text { for } \xi \in S_{\nu} .\end{array}\right.$

We set

$$
g_{\nu}(t, x)=\frac{\nu^{-1}\left|S_{\nu}\right|^{-1 / 2}}{\sqrt{2 \pi}^{m}} \int_{S_{\nu}} e^{i x \cdot \xi} \psi_{\nu}(t, \xi){\sqrt{1+|\xi|^{2}}}^{-p} d \xi^{8)}
$$

hence

$$
\hat{g}_{\nu}(t, \xi)= \begin{cases}\nu^{-1}\left|S_{\nu}\right|^{-1 / 2}{\sqrt{1+|\xi|^{2}}}^{-p} \psi_{\nu}(t, \xi) & \text { for } \xi \in S_{\nu}, \\ 0 & \text { for } \xi \bar{\epsilon} S_{\nu} .\end{cases}
$$

Then $\left\|g_{\nu}(t, x)\right\|_{p} \leqq \nu^{-1} \rightarrow 0$. But by (3.12), (3.13) and (3.14)

$$
\left\|v_{\nu}(T)\right\|_{p} \geqq 1 \text {. }
$$

This contradicts with $v_{\nu}(t)=u_{\varepsilon_{\nu}}(t)-u_{0}(t) \rightarrow 0$ in $H_{p, x}$ uniformly for $0 \leqq t$ $\leqq T$. The condition (3.5) is thus necessary.

Sufficiency of the conditions. Put $v_{\varepsilon}(t)=u_{\varepsilon}(t)-u_{0}(t)$, then $v_{s}(t)$ is given by

$$
\begin{aligned}
v_{\varepsilon}(t, x) & =\sum_{j=1}^{l} \frac{1}{\sqrt{2 \pi}^{m}} \int_{E^{m}} e^{i x \cdot \xi} Y_{j}(t, \xi, \varepsilon) \partial_{t}^{j-1}\left\{\hat{u}_{\varepsilon}(0, \xi)-\hat{u}_{0}(0, \xi)\right\} d \xi \\
& +\frac{1}{\sqrt{2 \pi}^{m}} \int_{E^{m}} e^{i x \cdot \xi}\left\{\int_{0}^{t} P_{l}(\varepsilon)^{-1} Y_{l}(t-\tau, \xi, \varepsilon) \hat{g}_{\varepsilon}(\tau, \xi) d \tau\right\} d \xi,
\end{aligned}
$$

where $g_{s}(t, x)=L_{\varepsilon}\left[u_{0}\right]-L_{0}\left[u_{0}\right]+f_{\varepsilon}(t)-f_{0}(t)$ and $\hat{g}_{s}(t, \xi)=\mathfrak{F}_{x}\left[g_{\varepsilon}(t, x)\right](\xi)$. From (3.4), (3.5) and (3.15) we can easily derive

$$
\left\|v_{\varepsilon}(t, x)\right\|_{p} \rightarrow 0 \text { uniformly for } 0 \leqq t \leqq T \text {, }
$$

if $\left\|\partial_{t}^{j-1}\left\{u_{\varepsilon}(0)-u_{0}(0)\right\}\right\|_{p} \rightarrow 0$ and $\left\|f_{\varepsilon}(t)-f_{0}(t)\right\|_{p} \rightarrow 0$ uniformly for $0 \leqq t \leqq T$. Q.E.D. 